\title{
Quantum Computing with Alkaline-Earth-Metal Atoms
}

\author{
Andrew J. Daley, ${ }^{1,2}$ Martin M. Boyd, ${ }^{3}$ Jun Ye, ${ }^{1,3}$ and Peter Zoller ${ }^{1,2}$ \\ ${ }^{1}$ California Institute of Technology, Pasadena, California 91125, USA \\ ${ }^{2}$ Institute for Theoretical Physics, University of Innsbruck, A-6020 Innsbruck, Austria and Institute for Quantum Optics \\ and Quantum Information of the Austrian Academy of Sciences, A-6020 Innsbruck, Austria \\ ${ }^{3}$ JILA, National Institute of Standards and Technology and University of Colorado, Boulder, Colorado 80309-0440, USA
}

(Received 14 August 2008; published 23 October 2008)

\begin{abstract}
We present a complete scheme for quantum information processing using the unique features of alkaline-earth-metal atoms. We show how two completely independent lattices can be formed for the ${ }^{1} S_{0}$ and ${ }^{3} P_{0}$ states, with one used as a storage lattice for qubits encoded on the nuclear spin, and the other as a transport lattice to move qubits and perform gate operations. We discuss how the ${ }^{3} P_{2}$ level can be used for addressing of individual qubits, and how collisional losses from metastable states can be used to perform gates via a lossy blockade mechanism.
\end{abstract}

DOI: 10.1103/PhysRevLett.101.170504

PACS numbers: 03.67.Lx, 42.50.-p

The first steps in implementing quantum information processing with neutral atoms have been taken in experiments with alkali-metal atoms. These have demonstrated basic building blocks including entangling gates with coherent collisions in optical lattices [1,2], Rydberg states [3], and cavity quantum electrodynamics [4,5], as well as high-fidelity register loading [6,7]. Challenges in the further development of neutral atom systems towards scalable quantum computing include single-qubit addressing, and the achievement of high-fidelity operations while avoiding decoherence, e.g., due to magnetic field fluctuations [2]. Alkaline-earth(-like) atoms, as developed in the context of optical clocks [8], and degenerate gases [9], offer unique and novel opportunities to address these challenges [10,11]. The advantages include the possibility to encode qubits in nuclear spin states, decoupled from the electronic state in both the ${ }^{1} S_{0}$ ground state and the very long-lived ${ }^{3} P_{0}$ metastable state on the clock transition [10]. We show below that these ground and excited states can be manipulated completely independently by laser light, allowing the construction of independent optical lattices for the two states. This leads to a quantum computing scenario where qubits are stored in long-lived states in a storage lattice (associated with the ${ }^{1} S_{0}$ ground state), and can be transferred with individual addressing to a transport lattice (associated with the ${ }^{3} P_{0}$ metastable state). This can be used to move qubits around, and perform high-fidelity entangling gate operations [see Fig. 1(a)], or also many such operations in parallel $[2,12]$. We discuss a complete quantum computing proposal in this context, with quantitative analysis for ${ }^{87} \mathrm{Sr}$ [8]. This toolbox of techniques developed here is also of immediate relevance for quantum simulation.

The details of our scheme are shown in Fig. 1. On the clock transition ${ }^{1} S_{0}{ }^{-3} P_{0}$, the nuclear spin essentially decouples from the electronic state. We can then encode qubits in nuclear spin states of different magnetic quantum number $m_{I}$ [e.g., for ${ }^{87} \mathrm{Sr}$ (with $I=9 / 2$ ), we can define $|0\rangle \equiv\left|{ }^{1} S_{0}, m_{I}=-9 / 2\right\rangle$, and $|1\rangle \equiv\left|{ }^{1} S_{0}, m_{I}=-7 / 2\right\rangle$, see Fig. 1(b)]. These states are very insensitive to magnetic field fluctuations. Because the ${ }^{1} S_{0}$ ground state and ${ }^{3} P_{0}$ metastable state (with lifetime $\sim 150 \mathrm{~s}$ for ${ }^{87} \mathrm{Sr}$ ) belong to different transition families and are separated by optical frequencies, we can search for two wavelengths where an optical field will generate an ac Stark shift for each of these states completely independently of the other, as shown in Fig. 2(a). In Figs. 2(b) and 2(c) we plot the polarizability of the ${ }^{1} S_{0}$ and ${ }^{3} P_{0}$ states of ${ }^{87} \mathrm{Sr}$ at different wavelengths computed from oscillator strengths in Ref. [13]. We see very clearly that at $627 \mathrm{~nm}$, the polarizability of ${ }^{3} P_{0}$ is zero because of canceling shifts of different signs from more a)
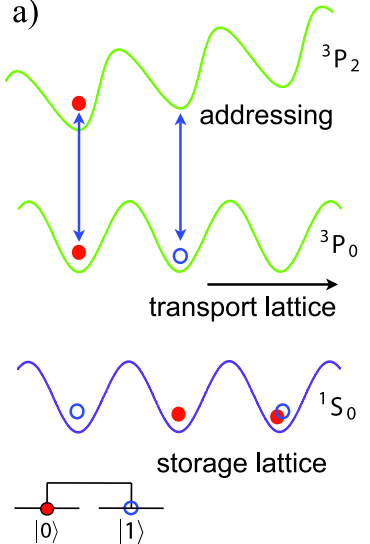

b)

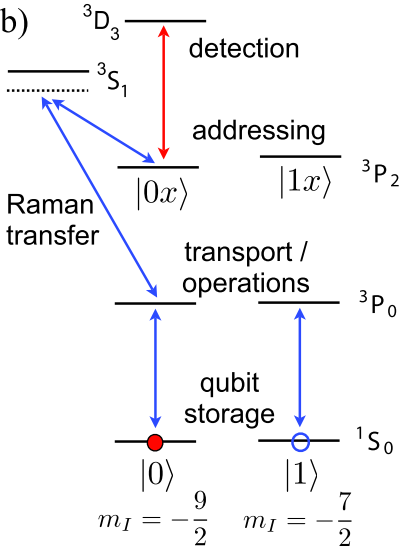

FIG. 1 (color online). Quantum computing with independent lattices: (a) Qubits in long-lived states in a storage lattice are transferred to a completely independent transport lattice for gate operations between distant qubits, or addressed individually by coupling to a level that is shifted by a gradient field. (b) This can be accomplished by encoding qubits in nuclear spin states, producing independent lattices for the ${ }^{1} S_{0}$ and ${ }^{3} P_{0}$ levels, and using ${ }^{3} P_{2}$ for individual addressing. 
a)

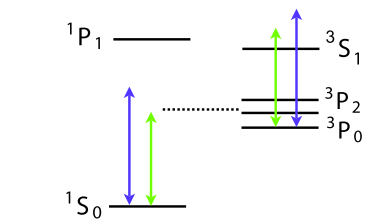

c)

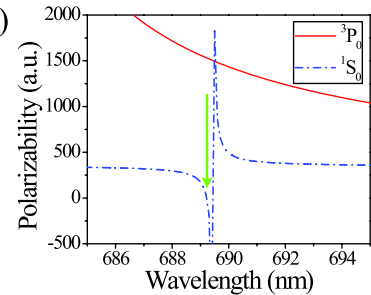

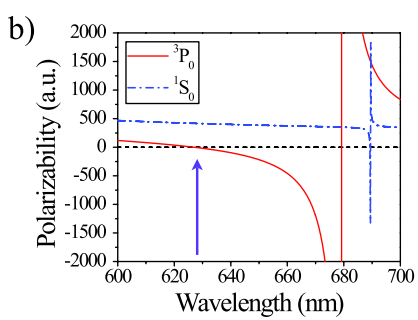

d)

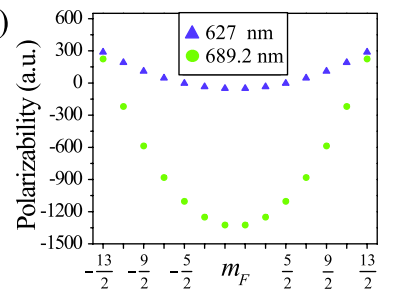

FIG. 2 (color online). (a) Energy level diagram showing how independent optical lattices can be produced for the ${ }^{1} S_{0}$ and ${ }^{3} P_{0}$ levels by finding wavelengths where the polarizability of each of the levels is zero and the other nonzero. (b) ac polarizability of ${ }^{1} S_{0}$ and ${ }^{3} P_{0}$ levels near $627 \mathrm{~nm}$. (c) ac polarizability of ${ }^{1} S_{0}$ and ${ }^{3} P_{0}$ levels near $689 \mathrm{~nm}$. (d) ac polarizability of different $m_{F}$ sublevels of the ${ }^{3} P_{2}, F=13 / 2$ hyperfine level for $\pi$-polarized light at 627 and $689.2 \mathrm{~nm}$.

highly excited triplet levels, while the polarizability of ${ }^{1} S_{0}$ is $\sim 430$ a.u.. Thus, we can form a deep optical lattice (where tunneling is negligible on experimental time scales) at a wavelength of $627 \mathrm{~nm}$ as a storage lattice for qubits, which will not affect the ${ }^{3} P_{0}$ states. Similarly, the polarizability of ${ }^{3} P_{0}$ at $689.2 \mathrm{~nm}$ is $\sim 1550$ a.u, whereas the polarizability of ${ }^{1} S_{0}$ is zero. This is largely because of the near-resonant coupling of ${ }^{1} S_{0}$ to ${ }^{3} P_{1}$, which is made possible without large spontaneous emission rates due to the narrow linewidth of ${ }^{3} P_{1}$. This lattice can be used for transport, and atoms in it will not be affected by the storage lattice. These lattices can be made to have the same spatial period by using angled beams in the case of the $627 \mathrm{~nm}$ light, so that the lattice period is increased to match that formed by counterpropagating beams at $689.2 \mathrm{~nm}$, and the depths can be made equal by using light of intensity $I_{0}$ for the storage and $\sim I_{0} / 4$ for the transport lattice, facilitating transfer of atoms between the two lattices. Gate operations can then be performed between distant sites by transferring atoms state-selectively into the transport lattice, and moving them to the appropriate distant site (see below for more details). This is somewhat reminiscent of the use of spindependent lattices for alkali atoms [2,12,14], where lattice lasers are tuned between fine-structure states, which can lead to large heating and decoherence from spontaneous emissions. Here, the lattices can be made completely independent by selection of the correct wavelengths. Note that while we illustrate our scheme in one dimension here, these ideas are generalizable to storage and transport lattices in $2 \mathrm{D}$ and $3 \mathrm{D}$.

An essential ingredient for general-purpose quantum information processing is the individual addressing of

qubits, both for readout and gate operations, which can be achieved in this system by coupling selectively to states in the long-lived ${ }^{3} P_{2}$ manifold. As shown schematically in Fig. 1 , we would transfer qubit states $|0\rangle$ and $|1\rangle$ to the ${ }^{3} P_{0}$ level (which can be done state-selectively in a large magnetic field due to the differential Zeeman shift of $109 \mathrm{~Hz} / \mathrm{G}$ between ${ }^{1} S_{0}$ and ${ }^{3} P_{0}$ ), and then selectively transfer them to additional readout levels $|0 x\rangle$ and $|1 x\rangle$ in the ${ }^{3} P_{2}$ level (e.g., for ${ }^{87} \mathrm{Sr}$ we could choose $|0 x\rangle \equiv \mid{ }^{3} P_{2}$, $\left.F=13 / 2, m_{F}=-13 / 2\right\rangle$ and $|1 x\rangle \equiv \mid{ }^{3} P_{2}, F=13 / 2$, $\left.m_{F}=-11 / 2\right\rangle$, where $F$ is the total angular momentum and $m_{F}$ the magnetic sublevel, and connect these states to the ${ }^{3} P_{0}$ level via off-resonant Raman coupling to a ${ }^{3} S_{1}$ level). The individual qubit selectivity can be based on a gradient magnetic field, as ${ }^{3} P_{2}$ is much more sensitive to magnetic fields ${ }^{3} P_{0}$ or ${ }^{1} S_{0}$. A gradient field of $100 \mathrm{G} / \mathrm{cm}$ will provide an energy gradient of $410 \mathrm{MHz} / \mathrm{cm}$ for $|0 x\rangle$ or an energy difference of about $15 \mathrm{kHz}$ between atoms in neighboring sites. In the same field the ${ }^{3} P_{0}$ level states will be shifted by $-m_{I} \times 1 \mathrm{~Hz}$ in neighboring sites, which again indicates the advantage of storing qubits on the nuclear spin states. This selectivity can be used to transfer atoms site-dependently to the transport lattice, or to read out qubits by transferring only the $|0\rangle$ state to ${ }^{3} P_{2}$, then making fluorescence measurements (e.g., using the cycling transition between the ${ }^{3} P_{2}, F=13 / 2$ and ${ }^{3} D_{3}$ manifold).

A necessary requirement here is that our states $|0 x\rangle$ and $|1 x\rangle$ are trapped in the combination of the storage and transport lattices (these will both provide ac Stark shifts for the ${ }^{3} P_{2}$ level). In Fig. 2(d) we plot the polarizability of all of the magnetic sublevels of ${ }^{3} P_{2}, F=13 / 2$ at our lattice wavelengths, and the large tensor shifts make certain $m_{F}$ levels suitable for trapping at the same locations as our qubit states. If the depths of the storage and transport lattices are chosen to be equal, then both the $|0 x\rangle$ level and the $|1 x\rangle$ will be trapped, in lattices about $2 / 3$ and $1 / 3$ of the depth of the storage lattice, respectively. The time scale for all transfer processes between lattices $\tau_{\text {trans }}$ is limited by the smallest trapping frequency $\omega_{t}$ (so that atoms are not coupled to excited motional states), and by the frequency shift $\omega_{e}$ between neighboring sites in the case of positionselective transfer, as $\tau_{\text {trans }} \gg \max \left(2 \pi / \omega_{t}, 2 \pi / \omega_{e}\right)$.

Single-qubit gates can be performed either by transferring atoms to the ${ }^{3} P_{0}$ level and then rotating the qubit states by directly applying Raman couplings, or alternatively with single-qubit addressability. This would involve using the ${ }^{3} P_{2}$ level in an intermediate step to transfer atoms position-selectively to the ${ }^{3} P_{0}$ level. Two-qubit gates are then performed using the transfer lattice. In particular, a phase gate between qubits in site $i$ and $j$ can be performed in a straightforward manner by: (i) transferring atoms in $|0\rangle$ on site $i$ (and $j$ ) to the transport lattice; (ii) moving the transport lattice relative to the storage lattice so that an atom that was originally in the $|0\rangle$ state on site $i$ would now be present at site $j$; (iii) generating a phase $\phi$ for the state conditioned on whether two atoms are on the same site 
[15]; and (iv) returning the atoms to their original position. In this protocol, if we express the state of the qubits in sites $i$ and $j$ in the basis $\left|q_{i}, q_{j}\right\rangle$, then the state $|0,1\rangle \rightarrow$ $\exp (i \phi)|0,1\rangle$, and all other states are unchanged. Many such phase gates can also be performed in parallel [12]. For example, cluster states [16] could be produced in a single operation entangling all atoms in neighboring sites.

The phase in step (iii) can be generated by an on-site collisional shift $U$ if the scattering length between two atoms in any combination of the ${ }^{1} S_{0}$ and ${ }^{3} P_{0}$ levels is significant. For $\mathrm{Sr}$ atoms the collisional interactions are normally weak, but could be increased using optical Feshbach resonances [17]. However, this also motivates the consideration of other gate schemes, especially blockade gates based on both coherent interactions and lossy channels in the ${ }^{3} P_{2}$ manifold. For sufficiently large on-site magnetic dipole-dipole interactions, which provide an energy shift $\Delta_{U}$ between ${ }^{3} P_{2}-{ }^{3} P_{2}$ and ${ }^{3} P_{0}{ }^{-3} P_{2}$ on-site collisional interactions, we can use a dipole blockade mechanism to produce a $\phi=\pi$ phase shift, as proposed, e.g., for Rydberg atoms [18]: (i) excite all $|0\rangle$ qubit states to an auxiliary level $|0 x\rangle$ with a $\pi$ pulse; (ii) couple all $|1\rangle$ qubit states to an auxiliary level $|1 x\rangle$ with a $2 \pi$ pulse at Rabi frequency $\Omega$, assuming that there is no collisional interaction between the $|0 x\rangle$ state and either $|1\rangle$ or $|1 x\rangle$. If the two atoms are on the same site the coupling is detuned by a frequency $\Delta_{U}$ and the transfer is blocked; (iii) return the $|0 x\rangle$ state to the $|0\rangle$ state with a $\pi$ pulse. This is shown schematically in Fig. 3(a) for the states $|01\rangle$ and $|10\rangle$. For a)
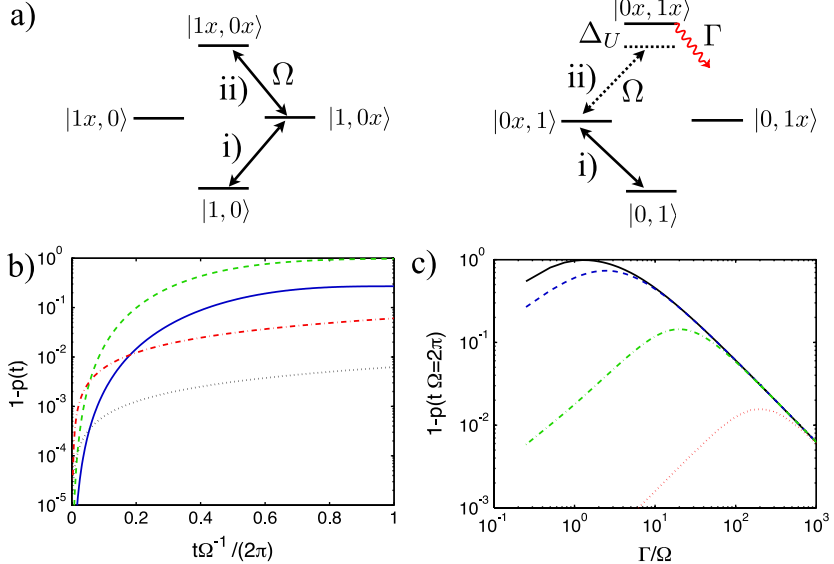

FIG. 3 (color online). (a) Two-qubits levels in a lossy blockade gate, contrasting behavior for the initial state $|1,0\rangle$, where the atoms are separated, and $|0,1\rangle$, where the atoms undergo collisions in the excited state on the same site. Atoms are (i) excited from the state $|0\rangle \rightarrow|0 x\rangle$, and then (ii) coupled from $|1\rangle \rightarrow|1 x\rangle$. The second process is blocked for the initial state $|0,1\rangle$ by elastic and inelastic collisional interactions. (b) Loss probability during step (ii) up to time $t$ with the initial state $|0 x, 1\rangle$ for $\Gamma / \Omega=1$ (solid line), 10 (dashed), 100 (dash-dotted), and 1000 (dotted), with $\Delta_{U}=0$. (b) Loss probability up to the gate completion time $\Omega t=2 \pi$ for $\Delta / \Omega=0$ (solid), 1 (dashed), 10 (dash-dotted), and 100 (dotted). two atoms not on the same site, two phases of $\pi$ are collected, so that $\left|q_{i}, q_{j}\right\rangle \rightarrow\left|q_{i}, q_{j}\right\rangle$. However, the state $|0,1\rangle$ collects only a phase $\phi=\pi$ due to the Rabi oscillation of only one atom, as the second transfer is blocked. In practice, the state $|0,1\rangle$ will collect a small additional phase $\phi \sim \Omega / \Delta_{U}$.

In addition to large elastic interactions, we expect large inelastic spin-flip losses in the ${ }^{3} P_{2}$ manifold, as discussed in Refs. [19], which could reduce the fidelity of the blockade gate. However, this loss can actually help us in producing the blockade effect, as large losses at a rate $\Gamma$ from a given level can also dynamically suppress occupation of that level. This is based on a similar mechanism to the recent observation of effective interactions induced by collisional losses in the motion of cold molecules [20], which is related to the continuous quantum Zeno effect. In the limit $\Delta_{U} \ll \Gamma$, one could even produce a gate based entirely on a lossy blockade mechanism. This is made possible because the energy change in the inelastic collision is larger than the lattice depth, so that we can assume that atoms are untrapped by the lattice, and coupled to a continuum of motional states. We can estimate the loss rates in the lattice based on the free-space values [19], assuming that the length scale on which the physics of the inelastic collision takes place is smaller than the confinement length in a lattice site, and that the collisions are thus unaffected by the presence of the lattice. The on-site loss rates from ${ }^{3} P_{2}$ could then reach values of the order of $\Gamma=2 \pi \times 20 \mathrm{kHz}$ for lattice densities of $10^{16} \mathrm{~cm}^{-3}$ [21].

In the presence of loss, the basic physics of the second step of the protocol, as illustrated in the right panel of Fig. 3(a) then reduces to a two level system, coupling the states $|g\rangle \equiv|0 x, 1\rangle$ and $|e\rangle \equiv|0 x, 1 x\rangle$. The non-Hermitian effective Hamiltonian describing the loss process is given by

$$
H=\frac{\Omega}{2}(|e\rangle\langle g|+| g\rangle\langle e|)+\left(-\Delta_{U}-i \frac{\Gamma}{2}\right)|e\rangle\langle e| .
$$

In the limit $\left|\Delta_{U}+i \Gamma / 2\right| \gg \Omega$, we can describe the time evolution of a system initially prepared in $|g\rangle$ in second order perturbation theory, giving the probability that no decay has occurred at short times $t$ as $p=\mathrm{e}^{-\Gamma_{\text {eff }} t}$, with $\Gamma_{\text {eff }} \approx \Omega^{2} \Gamma /\left[4\left(\Delta_{U}^{2}+\Gamma^{2} / 4\right)\right]$. For our lossy blockade gate the largest probability of loss occurs in the regime $\Gamma \gg$ $\Delta_{U}$, where the ratio of the loss time to the gate time (determined by $\Omega$ ) is given by $\tau_{\text {loss }} / \tau_{\text {gate }}=\Omega / \Gamma$. This will limit the fidelity of the lossy blockade gate to $1-$ $\Omega / \Gamma$, provided that there are no additional collisional shifts. If $\Delta_{U} \neq 0$, then the loss probability during the gate is decreased, as shown in Fig. 3(b), and the gate fidelity is correspondingly higher.

The fidelity of our gates and storage lifetime of our qubits are high due to the encoding of qubits in the nuclear spin states. For magnetic field fluctuations $\Delta B<10^{-3} \mathrm{G}$, the corresponding differential shift of the qubit states is $\Delta \omega_{B}<0.3 \mathrm{~Hz}$, as the Zeeman shift is $-185 \mathrm{~Hz} / \mathrm{G}$ in the 
${ }^{1} S_{0}$ level, and $-295 \mathrm{~Hz} / \mathrm{G}$ in the ${ }^{3} P_{0}$ level. This is suppressed by over 3 orders of magnitude compared with electron spin states. Relative intensity fluctuations in the storage and transport lattices will cause changes in the ground state energy of states in different lattices, but if this is controlled to one part in $10^{6}$, the relative shifts $\Delta \omega_{\text {intensity }}<0.05 \mathrm{~Hz}$. In the presence of both the storage and trapping lattices, each with a trapping frequency of $25 \mathrm{kHz}$, the spontaneous emission lifetimes of the various levels are $\sim 20 \mathrm{~s}$ for ${ }^{1} S_{0}, \sim 2 \mathrm{~s}$ for ${ }^{3} P_{0}$, and $\sim 1 \mathrm{~s}$ for ${ }^{3} P_{2}$. These constitute the largest source of decoherence during gate operations, but the associated time scales are much larger than the gate times, which in the worst case are limited by the trap frequency to be a few ms. We expect, therefore, that gate fidelities $\mathcal{F}>99 \%$ can be achieved in experiments. Similarly, collisional losses from metastable states, which occur only when two atoms are brought onto a single site, should play a small role except during lossy blockade gates, as discussed above. The collisional loss rates from ${ }^{3} P_{0}$ levels, which could play a role during the blockade gate operation are not yet known, however, for gate times on the order of $1 \mathrm{~ms}$, we require collisional stability of our atoms only for time scales of $100 \mathrm{~ms}$ in order to achieve gate fidelities $\mathcal{F}>99 \%$. On the other hand, if losses from the ${ }^{3} P_{0}$ are large, then these could also be directly used for a lossy blockade gate with the two atoms being coupled from ${ }^{1} S_{0}$ to ${ }^{3} P_{0}$.

As the isotopes of $\mathrm{Sr}$ or $\mathrm{Yb}$ with nonzero nuclear spin are fermionic, we have a substantial advantage in loading a quantum register with one atom per lattice site. If the lattice is ramped up adiabatically in the presence of a degenerate Fermi gas [9], a band insulator will form [7] provided that the temperature is smaller than the lattice band gap, and sites with missing atoms will typically be localized near the edges of any external trapping potential [22], leaving a regular array in the center of the trap. Moreover, because we have two internal states trapped by independent lattices, this system would be an ideal candidate for improvement of the quantum register by coherent filtering [23] or implementation of a fault-tolerant dissipative loading scheme [24].

This is a complete quantum computing proposal making use of the unique features of alkaline-earth atoms. In addition, the optical clock transition and nuclear spin states provide a natural basis for interfacing stationary (nuclear) and flying (photonic) qubits [5]. The clean realization of state-dependent lattices also opens a toolbox of techniques for quantum simulation [25], with such applications as implementation of spin models in optical lattices [26], or investigating dissipative dynamics with a reservoir gas coupled to atoms in an optical lattice [27].

We thank C. Greene, M. Lukin, A. Gorshkov, and L.-M. Duan for useful discussions. A. J. D. thanks the Institute for Quantum Information at Caltech for support, and A. J. D, J. Y, and P.Z. thank Caltech for hospitality. Work at JILA is supported by DARPA, NIST, and NSF, and work in Innsbruck is supported by the Austrian Science Foundation (FWF), and by the EU Network NAMEQUAM.

[1] M. Anderlini et al., Nature (London) 448, 452 (2007); S. Trotzky et al., Science 319, 295 (2008).

[2] O. Mandel et al., Phys. Rev. Lett. 91, 010407 (2003).

[3] E. Urban et al., arXiv:0805.0758; A. Browaeys et al. (unpublished).

[4] J. M. Raimond, M. Brune, and S. Haroche, Rev. Mod. Phys. 73, 565 (2001).

[5] H. J. Kimble, Nature (London) 453, 1023 (2008).

[6] M. Greiner et al., Nature (London) 415, 39 (2002); K. D. Nelson, X. Li, and D.S. Weiss, Nature Phys. 3, 556 (2007); D. Schrader et al., Phys. Rev. Lett. 93, 150501 (2004).

[7] M. Köhl et al., Phys. Rev. Lett. 94, 080403 (2005).

[8] J. Ye, H. J. Kimble, and H. Katori, Science 320, 1734 (2008); A. D. Ludlow et al., ibid. 319, 1805 (2008).

[9] Y. Takasu et al., Phys. Rev. Lett. 91, 040404 (2003); T. Fukuhara, Y. Takasu, M. Kumakura, and Y. Takahashi, ibid. 98, 030401 (2007).

[10] D. Hayes, P. S. Julienne, and I. H. Deutsch, Phys. Rev. Lett. 98, 070501 (2007); I. Reichenbach and I. H. Deutsch, ibid. 99, 123001 (2007).

[11] W. Yi, A. J. Daley, G. Pupillo, and P. Zoller, New J. Phys. 10, 073015 (2008); A. Gorshkov et al. (to be published).

[12] D. Jaksch et al., Phys. Rev. Lett. 82, 1975 (1999).

[13] S. G. Porsev, A. D. Ludlow, M. M. Boyd, and J. Ye, Phys. Rev. A 78, 032508 (2008).

[14] P. J. Lee et al., Phys. Rev. Lett. 99, 020402 (2007).

[15] Here it may be useful to transfer the $|0\rangle$ qubit state from site $j$ to an unoccupied site.

[16] R. Raussendorf and H. J. Briegel, Phys. Rev. Lett. 86, 5188 (2001).

[17] T. Zelevinsky et al., Phys. Rev. Lett. 96, 203201 (2006).

[18] D. Jaksch et al., Phys. Rev. Lett. 85, 2208 (2000).

[19] R. Santra and C. H. Greene, Phys. Rev. A 67, 062713 (2003); V. Kokoouline, R. Santra, and C. H. Greene, Phys. Rev. Lett. 90, 253201 (2003).

[20] N. Syassen et al., Science 320, 1329 (2008).

[21] Note that off-site loss contributions will be very small on the time scale of gate operations because we operate in deep lattices.

[22] L. Viverit, C. Menotti, T. Calarco, and A. Smerzi, Phys. Rev. Lett. 93, 110401 (2004).

[23] P. Rabl et al., Phys. Rev. Lett. 91, 110403 (2003).

[24] A. Griessner, A. J. Daley, D. Jaksch, and P. Zoller, Phys. Rev. A 72, 032332 (2005).

[25] I. Bloch, J. Dalibard, and W. Zwerger, arXiv:0704.3011v1.

[26] L.-M. Duan, E. Demler, and M. D. Lukin, Phys. Rev. Lett. 91, 090402 (2003).

[27] A. Griessner et al., Phys. Rev. Lett. 97, 220403 (2006); S. Diehl et al., arXiv:0803.1482v1. 